\title{
UN OUTIL DE PRISE DE DONNÉES SUR UNE IMAGE NUMÉRISÉE ET SON UTILITÉ DANS LES ÉTUDES RELATIVES AUX POISSONS : EXEMPLE D'UNE APPLICATION CONCRĖTE EN MORPHOMÉTRIE.
}

\author{
P. SAGNES
}

Laboratoire d'Ecologie des Eaux Douces et des Grands Fleuves, U.R.A. CNRS 1974, 43 bd du 11 novembre 1918, 69622 Villeurbanne Cedex, France.

\begin{abstract}
RÉSUMÉ
Une configuration informatique polyvalente en analyse d'images est présentée. L'accent est mis sur une application morphométrique par l'intermédiaire d'un exemple concret chez les poissons, d'où ressortent les principales qualités du dispositif, soit la précision et la rapidité de collecte et de stockage des données. Des applications possibles de cet outil à des domaines d'étude précis sont également suggérées.
\end{abstract}

\section{A TOOL FOR DATA COLLECTING ON A DIGITALIZED IMAGE AND ITS UTILITY IN FISH STUDIES : THE EXAMPLE OF A CONCRETE APPLICATION IN MORPHOMETRY.}

\section{SUMMARY}

A polyvalent data processing configuration for image analysis is presented. A morphometrical application on fish shows the main qualities of this apparatus : precision and rapidity of data collecting and data storage. Possible applications in definite fields are also suggested.

\section{INTRODUCTION}

Les études de croissance individuelle, la distinction entre plusieurs sous-populations ou variétés, l'écomorphologie, sont, entre autres, autant de domaines utilisant régulièrement des données morphométriques. Les exigences actuelles envers les outils permettant la collecte de telles données sont d'ordres divers. La première préoccupation des expérimentateurs est la précision des résultats. Dans la majorité des travaux relatifs aux poissons, une précision de 0,1 mm semble satisfaisante (SHARP et al., 1978 ; MAHON, 1984 ; MARTINEZ, 1984 ; WATSON et BALON, 1984 ; SCHAEFER et CAVENDER, 1986 ; ROBY et al., 1991 ; WINEMILLER, 1991 ; KARAKOUSIS et al., 1993). Pourtant, dans certains cas, il est légitime d'imaginer qu'une précision supérieure pourrait apporter des informations supplémentaires. L'utilisation de la loupe binoculaire ou du microscope peut répondre à cette attente, mais de telles méthodes de mesures directes présentent quelques inconvénients. Premièrement, des risques d'erreurs sont possibles lors de la lecture et lors de la retranscription des données en vue de leur stockage. Deuxièmement, la manipulation des animaux est souvent prolongée et nécessite alors que ceux-ci soient morts. La configuration informatique décrite ici, d'un accès facile, permet de s'affranchir de ces désagréments pour un coût relativement faible. Les performances et les avantages de cette méthode, basée sur la numérisation d'images, sont exposés par l'intermédiaire d'un exemple de prises de données morphométriques sur un poisson. II n'existe pas dans ce domaine de méthode couramment utilisée vraiment comparable : actuellement, seuls quelques points de repère anatomiques sont généralement digitalisés et stockés sous forme de coordonnées servant de base aux calculs ultérieurs (ROBY et al., 1991 ; WIMBERGER, 1993 ; BEEMAN et al., 1994). Cette technique n'est pas très souple, ne permettant que des calculs de longueurs (et non de surfaces, par exemple), et est surtout employée lors d'études utilisant les mesures en réseau décrites par STRAUSS et BOOKSTEIN (1982). 


\section{MATÉRIEL ET MÉTHODES}

Dans l'exemple développé plus loin, une exploitation confortable du matériel employé (configuration Apple ${ }^{\prime \prime}$ ) nécessite au minimum une mémoire vive de $6 \mathrm{Mo}$, la gestion de 256 couleurs et un moniteur 15 pouces. Les images que I'on souhaite traiter peuvent provenir de diverses sources telles qu'un magnétoscope, un lecteur photo CD ou une caméra vidéo (et donc indirectement une photo ou un négatif filmé(e) par cette caméra). Cette source transmet l'image à une carte d'extension nommée "Minichromax" (distribuée par Nautil ${ }^{\mathcal{C}}$ ) qui assure la numérisation de l'image, celle-ci pouvant être traitée de façon composite. Un logiciel associé du même nom (version 2.0), permet de modifier le contraste et la luminosité, de manière à obtenir une image optimale en fonction du type de données à recueillir. Un moniteur de contrôle s'avère alors utile pour affiner tous ces réglages précédant la numérisation définitive par la carte d'extension. L'image est ensuite enregistrée afin d'être réutilisée par l'intermédiaire du logiciel "Image" (distribué par NIH et dont la version 1.47 utilisée ici est en libre distribution) permettant la collecte et le stockage provisoire des données, après étalonnage grâce à une longueur connue numérisée conjointement au sujet principal. Le tableau de valeurs recueilli est alors immédiatement

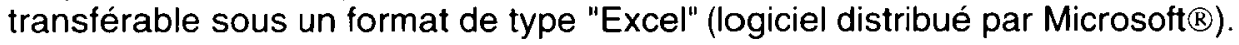

\section{APPLICATION À UNE ÉTUDE MORPHOMÉTRIQUE CHEZ LES POISSONS}

Dans l'exemple illustré ici, trois composantes (vue latérale, vue dorsale du poisson et bande millimétrée) sont regroupées sur une même image grâce aux possibilités de travail composite du logiciel "Minichromax" (figure 1). Les mesures de longueurs et de surfaces s'effectuent avec l'aide du logiciel "Image", respectivement en cliquant deux points ou en traçant des contours avec l'aide de la souris. Pour les poissons légèrement coudés (sous l'effet du conservateur, par exemple), ce logiciel permet une prise de mesures de longueurs cumulées par segments successifs.

\section{Incertitude sur les mesures}

La déformation des images engendrée par chaque objectif utilisé doit être contrôlée pour les différentes conditions de prises de vues (grossissement et diaphragme). Le logiciel "Image" autorise un très fort grossissement de l'image initialement numérisée, ce qui permet de travailler avec une précision égale au pixel (sans pour autant nécessiter un réétalonnage des mesures puisque la relation longueur-nombre de pixels reste la même). Sachant que l'erreur de localisation d'un point de repère est de l'ordre d'un pixel (dans le cas d'un point entre deux pixels par exemple), l'erreur relative maximale de mesure sur une variable dont la longueur est égale à $n$ pixels est donc (en pourcentage de cette longueur) de $2 / \mathrm{n}$. Pour obtenir la précision de $0,1 \mathrm{~mm}$ habituellement retenue, chaque pixel doit représenter au maximum $0,05 \mathrm{~mm}$. Sachant que, sous "Image", la longueur maximale affichée est de 736 pixels, la précision de $0,1 \mathrm{~mm}$ est obtenue pour une variable affichée plein écran mesurant $736 \times 0,05=36,8 \mathrm{~mm}$. Pour une variable supérieure à $36,8 \mathrm{~mm}$ la précision est toujours moindre, alors que pour une variable inférieure à $36,8 \mathrm{~mm}$ la précision peut être supérieure au dixième de millimètre (optimale lorsque la variable mesurée occupe la totalité de l'écran). La précision est donc définie lors de la numérisation de l'image et augmente proportionnellement au grossissement. A cette erreur essentiellement fonction du grossissement peut s'ajouter une erreur relative aux conditions de numérisation. Par exemple, certaines légères variations de l'angle de prise de vue peuvent augmenter la variabilité des résultats. Afin d'avoir une idée de la marge totale d'erreur sur des variables de différents ordres de grandeurs, des moyennes et leurs intervalles de confiance à $95 \%$ ont été établis en répétant trente fois sur un même individu les différentes manipulations de positionnement du poisson, de numérisation d'image et de mesures (tableau I). Dans cet exemple où un seul grossissement est utilisé, l'intervalle de confiance ne dépasse jamais $\pm 5 \%$. Cependant, une standardisation de la contention des poissons et des conditions de prises de vues pourrait être développée pour obtenir une répétabilité optimale des mesures et réduire au maximum la variabilité interexpérimentateur. 


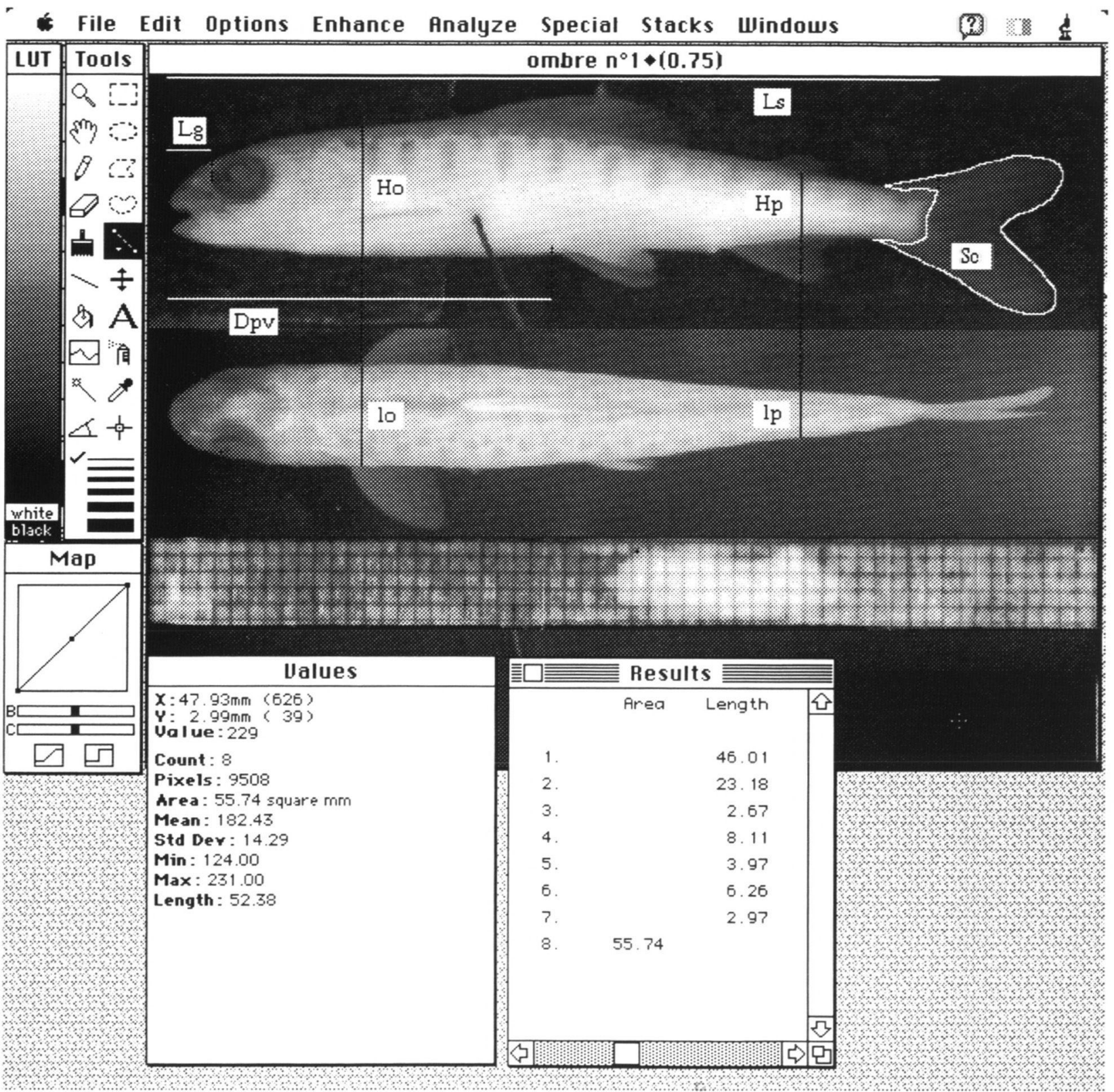


Figure 1 : Prise de mesures morphométriques sur un poisson : vue de l'écran sous le logiciel "Image" après numérisation de l'image par la carte d'extension et le logiciel associé "Minichromax".

L'image principale comporte une vue latérale, une vue dorsale du poisson et une bande de papier millimétré nécessaire à l'étalonnage. Les variables mesurées ici sont : 1 : Ls = longueur standard ; 2 : Dpv = longueur jusqu'à l'insertion des nageoires pelviennes ; $3: \mathbf{L g}=$ longueur jusqu'au globe oculaire ; $4: \mathrm{Ho}=$ hauteur au point le plus postérieur de l'opercule ; $5: \mathrm{Hp}$ = hauteur du pédoncule caudal au point d'insertion postérieure de la nageoire anale ; 6 : lo = largeur au point le plus postérieur de l'opercule ; $7:$ Ip = largeur du pédoncule caudal au niveau de la hauteur $\mathrm{Hp} ; 8: \mathrm{Sc}=$ surface de la nageoire caudale. Les autres fenêtres accessibles représentent : LUT $=$ palette des couleurs affichées $;$ Tools = outils de dessin et de mesure ; Map = réglage du contraste et de la luminosité de l'image principale ; Values = position du curseur et caractéristiques de la dernière mesure effectuée (valeur, nombre de pixels concernés, moyenne des densités de couleurs de ces pixels, etc...) ; Results = affichage des résultats, transférables directement sous un format de type "Excel". Les lignes de valeurs présentées ici correspondent respectivement aux mesures des variables énoncées ci-dessus (longueurs en $\mathrm{mm}$ et surface en $\mathrm{mm}^{2}$ ). Les mesures de longueurs et de surfaces sont respectivement prises en cliquant deux points sur l'image principale et en traçant à l'aide de la souris le contour de la surface désirée. Matériel optique utilisé pour cette prise de vue : caméra vidéo Kappa (CF 6/1), objectif Nikon f1.8/85 $\mathrm{mm}$ et bague allonge $5 \mathrm{~mm}$. Une telle image, non compactée, occupe un peu moins de $\mathbf{4 0 0}$ Ko de place mémoire.

Figure 1 : Collecting morphometrical data on a fish : a screen view of "Image" software after image digitalization by "minichromax" extension card and software.

The main image shows a lateral and a dorsal view of the fish and a piece of graph paper used for calibration. The variables presented are $: 1:$ Ls = standard length ; 2 : Dpv = length up to pelvic fin insertion ; $3: \mathrm{Lg}=$ length up to eye ; $4: \mathrm{Ho}=$ height at posterior point of gill cover ; $5: \mathrm{Hp}=$ caudal peduncle height at anal fin posterior insertion point ; $6: 10=$ width at posterior point of gill cover ; $7:$ Ip = caudal peduncle width corresponding to $\mathrm{Hp} ; 8$ : Sc = caudal fin area. The other windows are : LUT = palette of available colours ; Tools $=$ drawing and measuring tools $;$ Map = setting of contrast and brightness of the main image ; Values = cursor position and characteristics of the last measure (value, number of pixels concerned, mean of the colour density of these pixels, etc...) ; Results = display of results, immediately transferable as an "Excel" worksheet. Results presented here are those of the measurement of the above-mentioned variables (lengths in $\mathrm{mm}$ and area in $\mathrm{mm}^{2}$ ). Lengths are measured by clicking two points on the main image and areas by outlining the surface with the mouse. Optical equipment used in this example : a video camera (Kappa CF 6/1), a f1.8/85 mm Nikon lens and a $5 \mathrm{~mm}$ collar. The memory size occupied by such an uncompacted image is slightly less than $400 \mathrm{~K}$. 
Tableau I : Moyennes et intervalles de confiance à $95 \%$ pour des répétitions de mesures sur un même poisson. Les manipulations de numérisation et de mesures ont été répétées trente fois pour l'individu et les variables représentés sur la figure 1 . Les moyennes des longueurs sont données en millimètres et celle de la surface de la nageoire caudale (Sc) en millimètres carrés. Les intervalles de confiance à $95 \%$ ont été calculés en multipliant les écarts types par 1,96 (loi normale). La logique d'une meilleure précision pour les variables les plus grandes est quasiment respectée, excepté pour la variable Dpv pour laquelle la précision de localisation du point d'insertion des nageoires pelviennes variait selon les conditions d'éclairage du poisson pendant la numérisation.

Table I : Means and $95 \%$ confidence intervals in replicate measures on a fish. Digitalization and data collecting were repeated thirty times for variables on the fish presented in figure 1. Lengths means are expressed in millimetres and mean of caudal fin area (Sc) in square millimetres. The $95 \%$ confidence intervals were calculated by multiplying standard deviations by 1.96 (normal law). As expected, precision was higher for larger variables, except for Dpv owing to unsteady location of the pelvic fin insertion point according to illumination during digitalization.

\begin{tabular}{|c|cc|}
\hline Variables & \multicolumn{3}{|c|}{ Moyennes et intervalles à 95\% } \\
\hline Ls & $45,39 \pm 0,51$ & $( \pm 1,12 \%)$ \\
Dpv & $23,04 \pm 0,43$ & $( \pm 1,87 \%)$ \\
Ho & $8,02 \pm 0,1$ & $( \pm 1,25 \%)$ \\
lo & $6,09 \pm 0,21$ & $( \pm 3,45 \%)$ \\
Hp & $3,91 \pm 0,14$ & $( \pm 3,58 \%)$ \\
lp & $2,87 \pm 0,12$ & $( \pm 4,18 \%)$ \\
Lg & $2,63 \pm 0,13$ & $( \pm 4,94 \%)$ \\
Sc & $55,36 \pm 1,9$ & $( \pm 3,43 \%)$ \\
\hline
\end{tabular}

\section{Avantages de la méthode}

Travailler sur une image composite permet d'adapter le grossissement (et donc la précision) à la variable étudiée. Toutefois, si l'opérateur opte pour une image formée de différents grossissements, autant d'échelles sont à inclure dans cette image pour autant d'étalonnages précédant les mesures. Cette technique permet également d'augmenter considérablement la profondeur de champ pour les forts grossissements en modifiant progressivement la mise au point et en composant l'image étape par étape. Cet avantage, allié à celui de travailler sur une image fixe ne nécessitant plus aucune manipulation de l'animal, permet de réduire la variabilité des résultats et d'obtenir cette précision du dixième de millimètre parfois utopique, par exemple sous une loupe binoculaire munie d'un micromètre. De plus, le fait de travailler sur une image présente divers avantages par rapport à une méthode plus conventionnelle de mesures directes. Premièrement, les images peuvent être prises directement sur le terrain, en fin de pêche, ce qui permet de relâcher les poissons aussitôt et ainsi d'éviter une mortalité inutile ou certaines lésions possibles lors de manipulations prolongées. Deuxièmement, après l'acquisition d'une image numérisée optimale en laboratoire, les points de repères anatomiques nécessaires à la prise de mesures sont marqués grâce aux outils de dessins du logiciel "Image" (figure 1). Ce marquage permet une rapidité d'exécution importante lorsqu'un grand nombre de mesures doit être effectué. Troisièmement, le repérage de certaines variables dans l'espace est facilité par la composition de l'image numérisée permettant un affichage simultané de vues différentes d'un même individu. Dans notre exemple, les largeurs au niveau de l'opercule et du pédoncule caudal (variables lo et ip, figure 1) auraient été difficilement accessibles par une méthode classique de mesures directes. Enfin, le stockage sur support informatique de ces images "marquées" facilite une vérification rapide des mesures en cas de besoin. 


\section{Mesures directes ou numérisation d'image : quelle méthode utiliser ?}

Comparer ces méthodes dans le but de remplacer l'une par l'autre n'est pas envisageable. La prise de données sur une image numérisée est un nouvel outil, utile dans certains cas précis, et le choix entre les deux méthodes doit se faire en fonction de plusieurs critères. Par exemple, l'aspect pratique doit être pris en compte : l'espèce concernée est-elle trop fragile pour être transportée sans dommages au laboratoire ? Les individus doivent-ils être sauvegardés et relâchés rapidement pour éviter une perturbation de l'équilibre écologique du site ? Dans ce cas, une prise de vue appropriée sur le terrain et un traitement ultérieur des images est sans doute préférable. La précision désirée doit évidemment influencer le choix : le dixième de millimètre habituellement utilisé est obtenu seulement sur les variables inférieures à $36,8 \mathrm{~mm}$ dans le cas de la numérisation d'images mais cette précision peut être considérablement accrue en augmentant le grossissement lors de la prise de vue ; la numérisation pourra alors être retenue pour les petites variables, par exemple dans le cas d'œufs, d'alevins, ou de mesures anatomiques internes. II est également important d'évaluer le temps que prend la collecte de données en fonction du nombre de variables étudiées : dans la méthode décrite ici, quelques minutes sont nécessaires pour positionner correctement l'animal et numériser l'image dans de bonnes conditions, ce qui peut entraîner une perte de temps significative si peu de variables doivent être mesurées ; toutefois, la rapidité de collecte et le stockage immédiat des données compense en partie ce désavantage. Enfin, les avantages précédemment cités de la méthode de numérisation (localisation de variables interdépendantes et retour rapide aux images) peuvent également déterminer le choix.

\section{AUTRES APPLICATIONS POSSIBLES}

Outre les mesures morphométriques classiques, les nombreuses possibilités de la boîte à outils "Image" permettent de recueillir des données précises de natures variées dans de nombreux domaines d'étude. Pour rester dans le domaine piscicole, le calcul d'angles peut s'avérer utile en scalimétrie ; la surface de l'image occupée par une densité de couleur donnée peut être utilisée dans divers calculs automatiques de surfaces; le profil des densités de couleur sur un axe donné (après standardisation des conditions d'éclairement lors de la prise de vue et définition d'un protocole adapté), pourrait peut-être fournir des échelles de mesure dans l'appréciation de la smoltification de salmonidés ou de l'argenture de l'anguille, ou encore être utile pour la lecture de bandes électrophorétiques. Sans être aussi performant dans tous ces domaines que peuvent l'être des logiciels spécifiques, les nombreuses possibilités de l'association "Minichromax"-"Image" en font un excellent outil polyvalent.

\section{REMERCIEMENTS}

Ces travaux ont été effectués dans le cadre d'un Programme Interdisciplinaire de Recherche Environnement du CNRS (P.I.R.E.) concernant "les relations entre le poisson et son habitat". Je remercie tout particulièrement P. GAUDIN pour sa collaboration lors de l'acquisition et l'installation du matériel vidéo et informatique, ainsi que B. STATZNER pour ses critiques constructives précédant la rédaction finale de cette note.

\section{BIBLIOGRAPHIE}

BEEMAN J.W., RONDORF D.W., TILSON M.E., 1994. Assessing smoltification of juvenile spring chinook salmon (Oncorhynchus tshawytscha) using changes in body morphology. Can. J. Fish. aquat. Sci., 51, 836-844.

KARAKOUSIS Y., PEIOS C., ECONOMIDIS P.S., TRIANTAPHYLLIDIS C., 1993. Multivariate analysis of the morphological variability among Barbus peloponnesius (Cyprinidæ) populations from Greece and two populations of $B$. meridionalis meridionalis and B. meridionalis petenyi. Cybium, 17 (3), 229-240.

MAHON R., 1984. Divergent structure in fish taxocenes of north temperate streams. Can. J. Fish. aquat. Sci., 41, 330-350. 
MARTINEZ A.M., 1984. Identification of brook, brown, rainbow, and cutthroat trout larvæ. Trans. Am. Fish. Soc., 113, 252-259.

ROBY D., LAMBERT J.D., SÉVIGNY J.M., 1991. Morphometric and electrophoretic approaches to discrimination of capelin (Mallotus villosus) populations in the estuary and Gulf of St Lawrence. Can. J. Fish. aquat. Sci., 48, 2040-2050.

SCHAEFER S.A., CAVENDER T.M., 1986. Geographic variation and subspecific status of Notropis spilopterus (Pisces : Cyprinidæ). Copeia, 1, 122-130.

SHARP J.C., ABLE K.W., LEGGETT W.C., CARSCADDEN J.E., 1978. Utility of meristic and morphometric characters for identification of capelin (Mallotus villosus) stocks in Canadian Atlantic waters. J. Fish. Res. Board Can., 35, 124-130.

STRAUSS R.E., BOOKSTEIN F.L., 1982. The truss : body form reconstructions in morphometrics. Syst. Zool., 31 (2), 113-135.

WATSON D.J., BALON E.K., 1984. Ecomorphological analysis of fish taxocenes in rainforest streams of northern Borneo. J. Fish. Biol., 25, 371-384.

WIMBERGER P.H., 1993. Effects of vitamine C deficiency on body shape and skull osteology in Geophagus brasiliensis: implications for interpretations of morphological plasticity. Copeia, 2, 343-351.

WINEMILLER K.O., 1991. Ecomorphological diversification in lowland freshwater fish assemblages from five biotic regions. Ecol. Monog., 61 (4), 343-365. 